\title{
CHANGE DETECTION WITH SYNTHETIC APERTURE RADAR IMAGES BY WILCOXON STATISTIC LIKELIHOOD RATIO TEST
}

\author{
Vladimir A. Krylov ${ }^{1}$, Gabriele Moser ${ }^{2}$, Aurélie Voisin ${ }^{1}$, Sebastiano B. Serpico ${ }^{2}$ and Josiane Zerubia ${ }^{1}$ \\ ${ }^{1}$ Ayin team, INRIA, 2004 route des Lucioles, BP93, F-06902 Sophia Antipolis, Cedex, France \\ \{vladimir.krylov, aurelie.voisin, josiane.zerubia\}@inria.fr \\ ${ }^{2}$ Dept. of Biophys. and Elect. Eng. (DIBE), Univ. of Genoa, Via Opera Pia 11a, I-16145, Genoa, Italy \\ \{gabriele.moser, sebastiano.serpico\}@unige.it
}

\begin{abstract}
This paper considers change detection with multitemporal synthetic aperture radar (SAR) images. A novel statistical approach is developed that formulates the detection of changes between two coregistered SAR images acquired at different dates as a non-parametric local-window hypothesis testing problem. This approach takes into account the simultaneous testing of a large amount of similar hypotheses (i.e., one Wilcoxon test at each pixel of the image), and is based on a likelihood ratio test to obtain a detection map at the pixel level. Some encouraging detection results are obtained on XSAR and very high resolution COSMO-SkyMed images.
\end{abstract}

Index Terms- Change detection, synthetic aperture radar, hypothesis test, Wilcoxon statistic, likelihood ratio test

\section{INTRODUCTION}

Synthetic aperture radar (SAR) imagery [1] is one of the major data sources for remote sensing applications. Despite the fact that remotely sensed optical imagery can be interpreted more easily by a human operator, it can only work during daytime and is subjected to weather conditions. Therefore, modern SAR sensors represent an indispensable source of all-weather and 24-hour imagery with a fixed re-visit cycle at competitive high resolution. Two-date change detection from SAR images is a process that employs two SAR images acquired over the same geographical area with possibly the same (or close) acquisition characteristics at two different times to map the areas where changes occur between the two acquisition dates. The advantages of SAR systems often render SAR a more suitable imagery source than optical sensors for the change detection application.

The central disadvantage of the SAR imagery is given by an inherent multiplicative speckle noise, which restricts the direct application of optical-based change detection methods to SAR imagery. A variety of change detection approaches have been developed in the literature: some are SAR-specific whereas others are developed for optical imagery but still report acceptable results on the speckled SAR imagery. They include: despeckled or window-based image ratioing [1], explicit ratio distributions [2], fuzzy hidden Markov chains [3], scale-driven [4] and kernel-based [5] methods, statistical similarity measures [6].

In this paper our aim is to develop a non-parametric statistical change detection approach. The reason is that a wide variety of parametric probability density function (PDF) models is available for SAR imagery [1,7]. The methods in $[2,6]$ are developed for several PDF-models which brings to distinct results under different assumptions. To avoid this ambiguity we will not assume any specific PDF model for the statistics of SAR. We develop a modified hypothesis test which is based on the classical Wilcoxon two-sample test [8] that verifies whether one of two samples of independent observations tends to have larger values than the other. The choice of the Wilcoxon statistic as compared to the other available goodness-of-fit test statistics, such as, e.g., that of Cramér-von Mises' test [8], is a compromise solution to have simultaneously an analytically tractable asymptotic distribution (which is needed to formulate the likelihood ratio test) and a non-parametric testing procedure. Furthermore, the experimental validation demonstrated the adequacy of this statistic to the considered problem. To be able to take a decision at each pixel of the coregistered image pair we considered samples originating from the local windows centered in each pixel. Finally, we construct a likelihood ratio test on the image with Wilcoxon statistic values. This formulation allows to overcome the classical independency assumption for the Wilcoxon test which is violated (at least, locally) with the local window samples. The resulting technique is related to the statistical false discovery rate approach developed for "large-scale simultaneous hypothesis testing" problems [9], however the derivation and interpretation are different.

The remainder of the paper is organized as follows. In Sec. 2, we develop a novel Wilcoxon statistic likelihood ratio test for unsupervised multitemporal SAR change detection. In Sec. 3, we report experiments and comparisons of the developed method on XSAR and very high resolution COSMOSkyMed imagery. Finally, in Sec. 4, we draw conclusions. 


\section{CHANGE DETECTION METHOD}

The change detection consists of the following steps:

1) calculate Wilcoxon statistic $w(i, j)$ values by using (1);

2) estimate the null density $f_{0}(w)$ as defined in (2)-(3);

3 ) estimate the PDF $f_{W}(w)$ by using natural splines;

4) calculate likelihood ratio $\Lambda$ at each pixel as in (4);

5) report pixels with $\Lambda<T$ as changed.

The details are provided in the following subsections.

\subsection{Wilcoxon test}

Given two independent random variables $X$ and $Y$, the Wilcoxon rank-sum test [8] verifies the null hypothesis $\left\{H_{0}^{W}: \quad P(X<Y)=1 / 2\right\}$, with an alternative $\left\{H_{1}^{W}\right.$ : $P(X<Y) \neq 1 / 2\}$. Wilcoxon procedure tests whether the difference in central tendency between the two samples differs from zero, or, in other words, the samples comparative symmetry. It is consistent against a specific shift alternative $\left\{H_{1}: F_{X}(x) \equiv F_{Y}(x+a)\right\}$, where $F_{X}$ and $F_{Y}$ are the cumulative distribution functions of samples $X$ and $Y$, respectively, and $a$ is the shift. Therefore, we will interpret the test results as relevant to the first order shift characteristic, which is one of the relevant characteristics for the SAR imagery [8].

We will calculate the Wilcoxon statistic on samples coming from the local windows centered at the analyzed pixel coordinate on the two coregistered SAR images acquired at different dates. To calculate the the two-sample Wilcoxon statistic $W$ the observed samples $X_{i}$ and $Y_{j}, i, j=1, \ldots, N$, are concatenated into the sample $Z$ of size $2 N$, which is then ordered to obtain $Z_{(1)}<\cdots<Z_{(2 N)}$. Elements of the sample $X_{i}$ are positioned in $Z_{(k)}$ at places (ranks) $R_{1}, \ldots, R_{N}$. $W$ is then defined as the rank-sum $R=\sum_{i=1}^{N} R_{i}$. The standardized Wilcoxon statistic value is defined as

$$
W=\frac{\sum_{i=1}^{N} R_{i}-\frac{N(2 N+1)}{2}}{\sqrt{\frac{N^{2}(2 N+1)}{12}}}
$$

and converges to the standard normal distribution $N(0,1)$ [8] when $N \rightarrow \infty$ under independency assumptions. Thus, for large samples the critical values for the Wilcoxon test can be drawn from the normal approximation, that is classically employed when $N>20$ [8]. The rejection region of the test is two-sided, i.e., $H_{0}^{W}$ is rejected when $|W|>q$, where $q$ is a relevant quantile of the standard normal distribution.

Now one needs to adapt the Wilcoxon test to the considered problem which involves both the samples interdependencies ( $X$ and $Y$ are correlated as representing the same locations) and intra-dependencies (pixels inside both images are correlated because of the local spatial context). The textbook technique to address the inter-dependence is by considering the signed-rank sample Wilcoxon test modification [8]. However, this would assume a perfect (pixel-level) alignment (coregistration) of the images which is unfeasible. Furthermore, experiments performed with this test

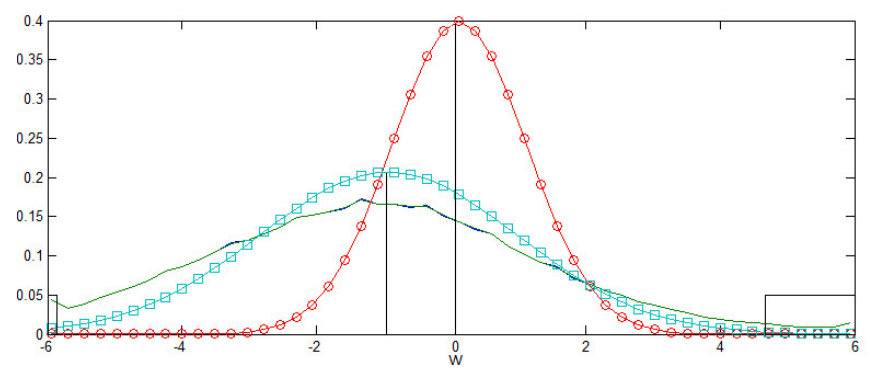

Fig. 1: Wilcoxon test on the COSMO-SkyMed image pair, 5x5 local-window: $f_{W}$ normalized histogram splines [spline approximation] (line), theoretical null PDF $N(0,1)$ (circles), and $f_{0}=N\left(-1.06,1.93^{2}\right)$ (squares). The areas in rectangles correspond to the detection with the proposed method.

modification demonstrated an appreciable deterioration of the detection accuracy. Another widely used technique to obtain empirical p-values, given by bootstrap [8], cannot be employed because of the intra-dependencies. Therefore, we adopt the standard Wilcoxon test defined in (1) to address the dependency scenario at hand.

\subsection{Wilcoxon statistic distribution}

The considered problem belongs to the so-called "large-scale simultaneous hypothesis testing" problems [9]: we perform hundreds of thousands simultaneous tests on the same image (one Wilcoxon test per pixel). Each two-sample Wilcoxon statistic $W$ should follow the standard normal distribution under the independency assumption. In our problem, however, the statistic values $W$ are dependent, at the very least, at neighboring locations. Therefore, the use of the theoretical null distribution $N(0,1)$ would bring to erroneous results. To deal with this problem we capitalize on the knowledge of the Wilcoxon statistic histogram available after calculating the statistic value at each image pixel to learn more about the $W$ distribution under the $H_{0}^{W}$ assumption.

To estimate the distribution of the Wilcoxon statistic we adopt the following strategy: we keep the same family of the theoretical null distribution (i.e., normal) but modify its parameters so as to fit better the part of the observed histogram density that corresponds to the unchanged areas. More specifically, we estimate the distribution mean $\mu$ and variance $\sigma^{2}$ as the sample $2 \alpha$-trimmed moments:

$$
\hat{\mu}=\frac{1}{\beta} \sum_{i=\alpha N}^{(1-\alpha) N} W_{(i)}, \hat{\sigma}^{2}=\frac{1}{\beta-1} \sum_{i=\alpha N}^{(1-\alpha) N}\left[W_{(i)}-\hat{\mu}\right]^{2},
$$

where $\alpha$ is chosen so that $\alpha N$ is integer, $\beta=(1-2 \alpha) N$. The the order statistic $W_{(1)}<\cdots<W_{(N)}$ is obtained from $W$ by removing the $\alpha N$ smallest and $\alpha N$ largest values. Thus, we model the distribution of $W$ under $H_{0}^{W}$ as

$$
f_{0}(w)=N\left(\hat{\mu}, \hat{\sigma}^{2}\right) .
$$




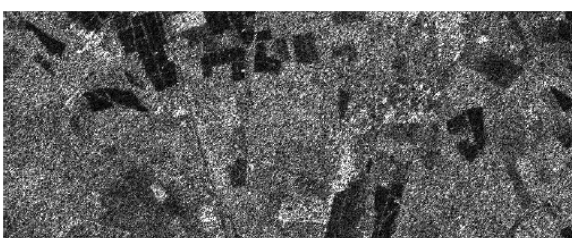

(a) XSAR image, April 161994

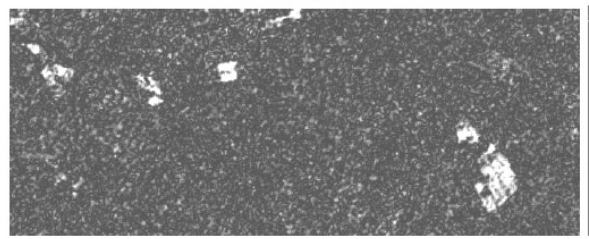

(d) Image ratio, $5 \times 5$ window

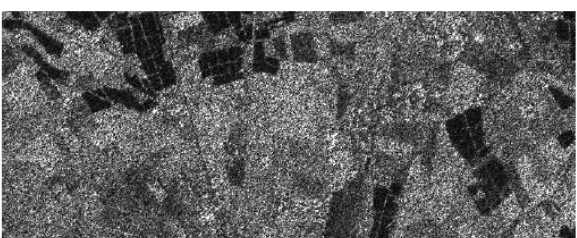

(b) XSAR image, April 181994

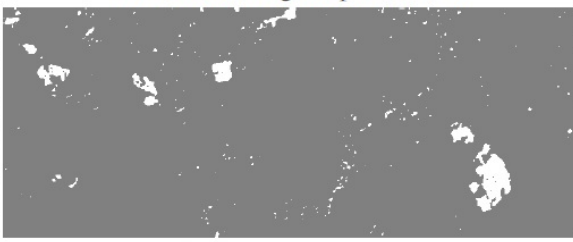

(e) LN-GKIT detection method

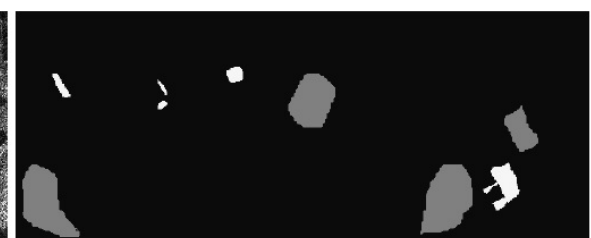

(c) Change reference map

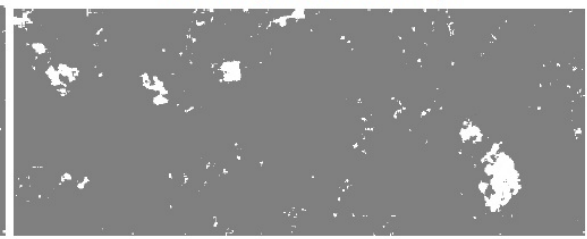

(f) Proposed method, $5 \times 5$ window

Fig. 2: Coregistered XSAR images acquired on (a) April 16, 1994 and (b) April 18, 1994 over an agricultural area near Pavia, Italy, (c) non-exhaustive change reference map (grey - no change, white - change), and detection results: (d) 5x5 window-based image ratio [1], (e) LN-GKIT detection after despeckling [2], (f) proposed method with 5x5 window.

An example of such empirical fit is shown in Fig. 1 for the Wilcoxon statistics based on the images in Fig. 3 (a), (b). This approach works under an assumption that the changed pixels are those that report Wilcoxon statistic values that lie far from its center. More specifically, we expect the changed pixels to demonstrate a statistically different behavior that results in reporting significantly larger values of the Wilcoxon statistic.

\subsection{Likelihood ratio test}

At this stage the initial change detection is reduced to Wilcoxon statistic image analysis. At each pixel location $(i, j)$ we proceed by implementing a likelihood ratio test [8] with the $\left\{H_{0}^{L R T}: W_{(i, j)} \sim f_{0}\right\}$, where $f_{0}(w)$ is defined in (3), and the noninformative alternative $\left\{H_{1}^{L R T}: W_{(i, j)} \sim\right.$ $\left.f_{W}\right\}$, where $f_{W}(w)$ is the observed PDF (histogram) of the Wilcoxon statistic on all the image pixels. Correspondingly, the decision is based on the following statistic [8]:

$$
\Lambda=f_{0}(w) / f_{W}(w)
$$

Unfortunately, for the considered problem, no distribution is available for $\Lambda$ [8]. Therefore, we will restrict the analysis of $\Lambda$ to simple one-side thresholding since the critical area is left-sided $(0, T)$. The threshold value $T$ is proportional to the amount of detection (rejection area of $H_{0}^{L R T}$ ). For a more detailed analysis see [9], where $\Lambda$ is derived under the false discovery rate formalism.

\section{EXPERIMENTAL RESULTS}

The developed detection procedure has three parameters: local window size SxS, the trimming proportion $\alpha$ and the threshold $T$. The window size $\mathrm{S}$ should not be less than 5 to ensure the normal approximation, yet not too large to avoid excessive oversmoothing. The trimming can be modified to accommodate scenarios when most of the changes are localized in one of the tails of the histogram. To estimate $f_{W}(w)$ we have employed the natural spline with ten degrees of freedom to the $\mathrm{W}$ statistic histogram. For the likelihood ratio thresholding we will follow the somewhat empirical choice of [9] and set $T=0.1$.

We validate the developed change detection approach on the following two datasets:

- shuttle-born XSAR image pair acquired on April 16, 1994 and April 18, 1994 over an agricultural area near Pavia, Italy, approx. 15 m resolution, four-look, $700 \times 280$ pixels, Fig. 2 . - space-born COSMO-SkyMed image pair (CASI, 2010) acquired on 22 January, 2010 and 22 February, 2010 in the port area of Port-au-Prince, Haiti, SpotLight mode, $1 \mathrm{~m}$ resolution, single-look, $2500 \times 2500$ pixels, Fig. 3 .

In all the experiments a two-sided $\alpha=10 \%$ trimming was performed for $f_{0}(w)$ PDF estimation, the local window size is set to $5 \times 5$, i.e., $N=25$, and the detection threshold is $T=0.1$.

On XSAR dataset we validate the obtained change detection accuracy by employing a partial ground truth, see Fig. 2(c). We present comparisons with the benchmark image ratio (local window-based) thresholding result [1] in Fig. 2(d), and the result of Lognormal-GKIT model after despeckling [2] in Fig. 2(e). For the image ratio the threshold was set to obtain the same detection of $3 \%$ of pixels. The performances are summarized in Table 1 . In order to estimate the detector's robustness to speckle, we have run the proposed method on a despeckled pair of images by applying a $7 \times 7$ Gamma-MAP filter. The reported results are very similar and we present only the numeric accuracies of this experiment in Table 1.

The second experiment is performed on a high resolution COSMO-SkyMed dataset. No reference map is available, 
Table 1: Change detection performances reported on the XSAR dataset.

\begin{tabular}{|l||c|c|c|}
\hline Method & $\begin{array}{c}\text { False } \\
\text { positive }\end{array}$ & $\begin{array}{c}\text { True } \\
\text { positive }\end{array}$ & $\begin{array}{c}\text { Detection } \\
\text { amount }\end{array}$ \\
\hline \hline Window ratio [1] & $5.8 \%$ & $79.1 \%$ & $3 \%$ \\
\hline LN-GKIT [2] & $0.38 \%$ & $87.2 \%$ & $2.84 \%$ \\
\hline Proposed & $0.56 \%$ & $92.6 \%$ & $3.55 \%$ \\
\hline Proposed + despeckling & $0.48 \%$ & $93.1 \%$ & $3.82 \%$ \\
\hline
\end{tabular}
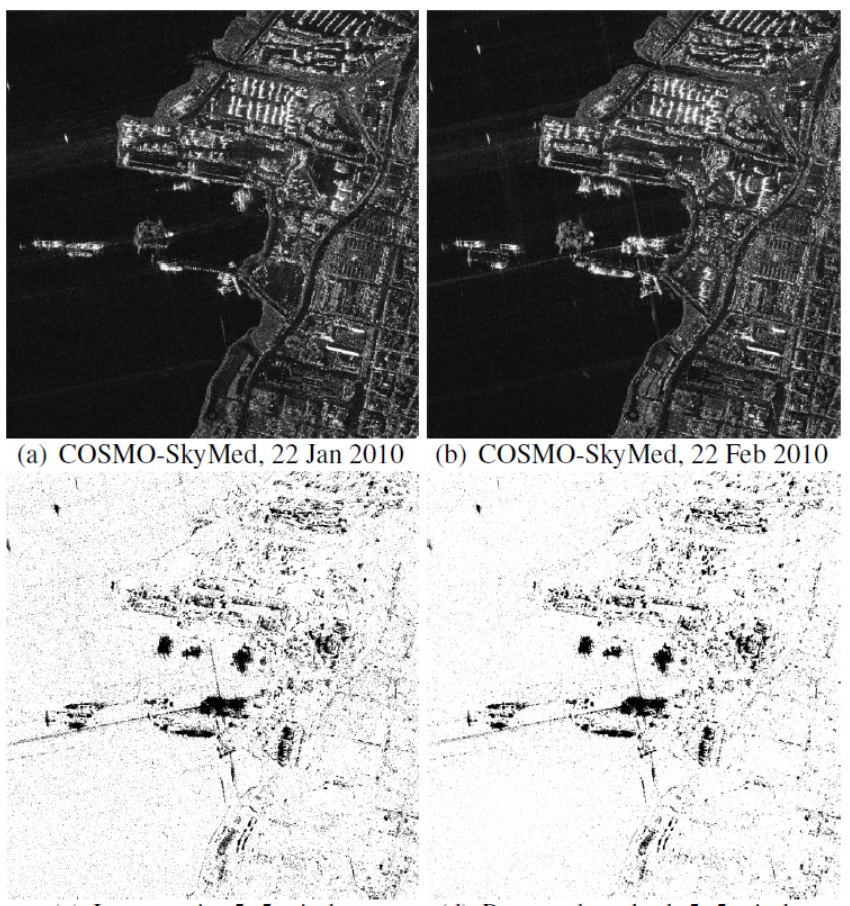

(c) Image ratio, $5 \times 5$ window
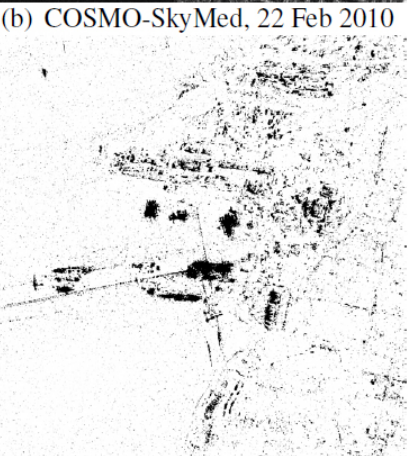

(d) Proposed method, $5 \times 5$ window

Fig. 3: Coregistered COSMO-SkyMed images (C)ASI) acquired on (a) January 22, 2010 and (b) February 22, 2010 over Port-au-Prince, Haiti, and detection results: (c) $5 \times 5$ window-based image ratio [1] with $10 \%$ detection, and the (d) proposed method with $5 \times 5$ window and $7.4 \%$ detection.

and, therefore, no performance estimates are possible. We present a comparison to the window-based image ratioing method, see Fig. 3, to demonstrate a visually less noisy behavior of the detection map reported by the proposed method.

\section{CONCLUSIONS}

In this paper we have developed a novel non-parametric Wilcoxon statistic-based likelihood ratio test for unsupervised SAR change detection. The advantages of the developed approach are the absence of distribution assumptions, the experimentally validated robustness to speckle, and the low computational complexity. The main directions of further research include the automatic estimation of parameters $(\alpha, T)$, multiresolution improvements [4], and validation with other types of imagery.

\section{ACKNOWLEDGMENT}

The authors would like to thank Prof. P. Gamba (University of Pavia, Italy) for providing the XSAR dataset, and the Italian Space Agency for providing the COSMO-SkyMed (CSK $\AA$ ) dataset of Haiti (COSMO-SkyMed Product - (C)ASI - Agenzia Spaziale Italiana - 2010. All Rights Reserved) in the framework of the project "Development and validation of multitemporal image analysis methodologies for multirisk monitoring of critical structures and infrastructures (2010-2012)".

\section{REFERENCES}

[1] C. Oliver and S. Quegan, Understanding Synthetic Aperture Radar Images, Artech House, Norwood, 1998.

[2] G. Moser and S. B. Serpico, "Generalized minimumerror thresholding for unsupervised change detection from SAR amplitude imagery," IEEE Trans. Geosci. Remote Sens., vol. 44, no. 10, pp. 2972-2982, 2006.

[3] C. Carincotte, S. Derrode, and S. Bourennane, "Unsupervised change detection on SAR images using fuzzy hidden Markov chains," IEEE Trans. Geosci. Remote Sens., vol. 44, no. 2, pp. 432-441, 2006.

[4] T. Celik, "A Bayesian approach to unsupervised multiscale change detection in synthetic aperture radar images," Signal Process., vol. 90, pp. 1471-1485, 2010.

[5] G. Camps-Valls, L. Gomez-Chova, J. Munoz-Mari, M. Martinez-Ramon, and J. L. Rojo-Alvarez, "Kernelbased framework for multi-temporal and multi-source remote sensing data classification and change detection," IEEE Trans. Geosci. Remote Sens., vol. 46, no. 6, pp. 1822-1835, 2008.

[6] J. Inglada and G. Mercier, "A new statistical similarity measure for change detection in multitemporal SAR images and its extension to multiscale change analysis," IEEE Trans. Geosci. Remote Sens., vol. 45, no. 5, pp. 1432-1445, 2007.

[7] V. A. Krylov, G. Moser, S. B. Serpico, and J. Zerubia, "Enhanced dictionary-based SAR amplitude distribution estimation and its validation with very high-resolution data," IEEE Geosci. Remote Sens. Lett., vol. 8, no. 1, pp. 148-152, 2011.

[8] E. L. Lehmann and J. P. Romano, Testing statistical hypotheses, Springer, New York, 3rd edition, 2005.

[9] B. Efron, "Large-scale simultaneous hypothesis testing: The choice of a null hypothesis," J. Am. Stat. Assoc., vol. 99, no. 465, pp. 96-104, 2004. 